\title{
Pierre Caspard, Jean-Noël Luc et Philippe Savoie (dir.) \\ Lycées, lycéens, lycéennes. Deux siècles d'histoire.
}

Lyon : Institut national de recherche pédagogique, 2005, 501 pages.

\author{
Ollivier Hubert \\ Université de Montréal et Centre interuniversitaire d'études québécoises-Laval
}

Cet ouvrage est issu d'un colloque organisé à la Sorbonne en 2002 à l'occasion du bicentenaire des lycées en France. L'excellente introduction de Jean-Noël Luc permet de remettre à l'esprit les paramètres fondamentaux de leur histoire, depuis la loi du 11 floréal an X jusqu'aux incertitudes actuelles. Le lycée demeure, jusque dans les années $1930 \mathrm{au}$ moins, une machine à produire ou reproduire les élites (intellectuelles ? sociales ? politiques? numériques ?; ce point demeure assez obscur) de la nation, puisque moins de $4 \%$ des Français de sexe masculin en sortent avec un diplôme. Il s'inscrit d'abord dans la continuation du collège d'Ancien Régime et s'impose progressivement face au secteur privé, principalement catholique. À la fin $\mathrm{XIX}^{\mathrm{e}}$ siècle, la diversification des filières et les débuts d'un réseau pour les filles préparent une démocratisation qui s’opère, par poussées, au long du siècle suivant : c'est une évolution qui change radicalement la nature même du projet, de sorte que les études présentées dans ce collectif pourraient être scindées en deux groupes selon cette ligne de partage : avant ou après la massification. Luc donne encore une utile présentation de la très riche historiographie qui concerne ces établissements : les approches générales, institutionnelles et politiques, puis sociales, toujours pertinentes évidemment, s'enrichissent d'études plus précises sur tel ou tel aspect. Le lycée est donc un objet d'histoire dont le potentiel est bien loin d'être épuisé, mais à propos duquel la recherche est bien développée.

La première partie de l'ouvrage traite du lycée dans son rapport à l'identité politique de la France. Une solide contribution de Philippe Savoie permet de faire le point sur le premier siècle du lycée (un pendant de cet article pour le $\mathrm{XX}^{\mathrm{e}}$ siècle fait 
malheureusement défaut) au cours duquel le modèle du collège humaniste, d'abord structurant, est progressivement abandonné pour faire place à un système différent, avec l'autonomisation des disciplines, le cours magistral, la mobilité du corps professoral et, finalement, la poussée de l'externat et la remise en cause du latin. Les contributions suivantes permettent de plonger au cœur des rapports entre l'institution des lycées et l'acculturation au républicanisme. Les textes de Marie-Madeleine Compère sur les « boursiers nationaux » et de Julien Vasquez, qui étudie l'offre et la demande à l'échelle locale, illustrent bien un processus d'implantation assez lent au long du XIX siècle, mais qui au bout du compte est une réussite. Malgré les embûches, le lycée a un rôle clef dans l'élévation du niveau des études et stimule l'unité nationale. Patrick Cabanel, expliquant avec raffinement la surreprésentation des enfants issus des minorités religieuses, permet de toucher du doigt la longue méfiance des vieilles élites catholiques à l'égard des établissements de la République. La question religieuse est du reste un thème important du recueil (Jean-Luc Marais sur la figure de l'aumônier, Frédéric Abécassis sur les lycées français, entre autres) et permet de bien voir comment le type français de laïcité est pour une part le produit du lycée, qui offre aux élites un terrain de coexistence. Sur le plan des hiérarchies sociales en revanche, la volonté de distinction a sans doute contribué à figer le système dans un modèle éducatif abstrait, déconnecté de la vie collective et même économique, malgré les critiques continues des progressistes. Jean-Michel Chapoulie montre de fait que la démocratisation et le passage au lycée de masse au milieu du $\mathrm{XX}^{\mathrm{e}}$ siècle ne sont pas séparables d'une réforme de l'enseignement dispensé.

La deuxième partie de l'ouvrage regroupe des contributions qui évoquent l'évolution des enseignements. Les études montrent bien l'importance cruciale de l'histoire des matières scolaires pour l'épistémologie et l'histoire des sciences, car l'ordre secondaire offre aux diverses branches du savoir une source de légitimation sociale, un terrain d'exercice et une réserve imposante de praticiens qui ne manquent pas de les penser et de les transformer. On est particulièrement fasciné par le texte d'Annie Bruter, enrichi par celui de Philippe Marchand, sur la lente autonomisation et les usages politiques, sociaux et culturels de l'histoire dans le premier XIX ${ }^{\mathrm{e}}$ siècle. Il est bon de sortir du cadre qui réduit trop souvent l'enseignement de cette matière au seul endoctrinement nationaliste. C'est de la rhétorique aussi qu'émerge l'enseignement de la littérature moderne étudié par Martine Jey. Les autres études (les sciences avec Jean-Yves Dupont, les mathématiques avec Renaud D'Enfert) montrent bien à quel point la relative souveraineté des ordres d'enseignement, les perpétuelles refontes de filières, les attentes du supérieur, constituent des éléments à prendre en considération. Mais la tension fondamentale se situe entre la volonté de plonger les jeunesses élitaires dans l'actualité du monde, et de leur donner ainsi les outils d'une critique de l'ordre, et celle de les fondre dans la matrice de la tradition. C'est donc le politique qui demeure à la surface comme au fond des choses, et avec une remarquable continuité : de ses origines jusqu’à aujourd'hui sans doute, la question du rapport de la jeunesse au monde reste débattue (Ludivine Bantigny l'évoque avec clarté pour les années 1950). Le conservatisme n'est pas seulement le produit d'une volonté cependant, comme le montre Évelyne Hery 
lorsqu'elle étudie l'effet de résistance à l'égard des réformes pédagogiques produit par les habitudes d'enseignants qui y voient une remise en question de leur identité.

La troisième partie, "Sociabilité, représentations et pratiques collectives ", est la plus courte et relève d'approches plus récentes. Il reste beaucoup à faire à propos des rapports entre les lycées et la construction culturelle des identités sociales. La question du genre est pour ainsi dire absente. Anne-Marie Sonh signe un texte remarquable sur l'idée de " jeunesse " dans les années 1960, à partir notamment de la correspondance adressée à une animatrice de radio : elle montre bien que parents (surtout les mères) et enfants ne sont pas dupes à propos de la "démocratisation ", même si beaucoup se jettent tout de même, avec angoisse, dans la course. On comprend alors, avec Robi Morder, que les revendications politiques des lycéens, d'abord bourgeoisement contestataires, glissent dans les années 1980 vers une défense de l'idéal du lycée pour tous. Car on soupçonne bien, quoiqu'aucun texte ne traite la question de front, qu'il y a lycée et lycée, et que les effets secondaires de la massification sont plus vivement ressentis dans les milieux moins favorisés que dans les quartiers des centres-villes. Trois autres contributions font une analyse de l'identité professorale. Il ne s'agit nullement d'une question indépendante de la crise que nous venons d'évoquer : les professeurs de philosophie (Bruno Poucet), par exemple, moulés par les concours de recrutement (Yves Verneuil) et distraits par les luttes intestines (André D. Robert), savent-ils toucher l'esprit et le cour des jeunes issus des milieux populaires et des classes moyennes inférieures ? La réponse passera peut-être par un élargissement de la sociologie du corps professoral.

La quatrième partie, "Ordre et changement dans l'administration des lycées ", nous plonge dans une problématique plus ancienne, bien que plus que jamais d'actualité, celle du " contrôle ", mais avec la volonté de briser le stéréotype du "lycée-caserne ", pourtant imposé par les souvenirs des anciens. Car tout le monde n'est pas fait pour les moules! L'architecture est étudiée par Marc Le Cœur qui fait comprendre les écarts entre les principes, édictés dès les origines (commodité, simplicité, propreté et ségrégation), et le concret de bâtisses souvent héritées. Petit à petit, le rationalisme impose une norme que l'on cherche maintenant à dépasser (Antoine Prost). L'approche est riche et demande développement, par exemple par l'étude de la manière dont les corps juvéniles se meuvent à l'intérieur de ces espaces imaginés. Sujet classique, la discipline est abordée par Jean-Claude Caron sous l'angle large et pertinent de l'imposition d'un ordre libéral au milieu du XIX ${ }^{\mathrm{e}}$ siècle. Le regard analytique révèle la complexité du problème qui fait, malgré tout, inexorablement penser à une réflexion sur le carcéral : la différence ici, toutefois, c'est le fractionnement de l'autorité entre le père et le directeur. Comme chez Foucault, l'humanisation apparente des peines n'est que la marque d'une société au fond plus contrôlante. Les internats sont passés mode vers la fin du XIX ${ }^{\mathrm{e}}$ siècle (Patrick Clastres, Jean-François Condette) : c'est le résultat de mutations conjuguées, parmi lesquelles domine l'émergence d'une « nouvelle sensibilité parentale " qui bouleverse tout. Plus généralement, la transition vers la société industrielle et démocratique aboutit à la réforme qui abolit (sur le papier) la distinction entre le classique et le moderne. 
Le recueil porte la marque du très dynamique Service d'histoire de l'éducation de l'Institut national de recherche pédagogique. La diversité des disciplines engagées, ainsi que le choix de privilégier la publication de résultats de recherche, même parfois préliminaires, plutôt que de textes relevant de la synthèse, font un tout de haute qualité. Le projet permet de plus à ses promoteurs d'identifier une piste sous-exploitée, ce qui est toujours bien précieux : le lycéen lui-même (et plus encore la lycéenne), qui est le grand absent, comme le note Pierre Caspard en conclusion. 